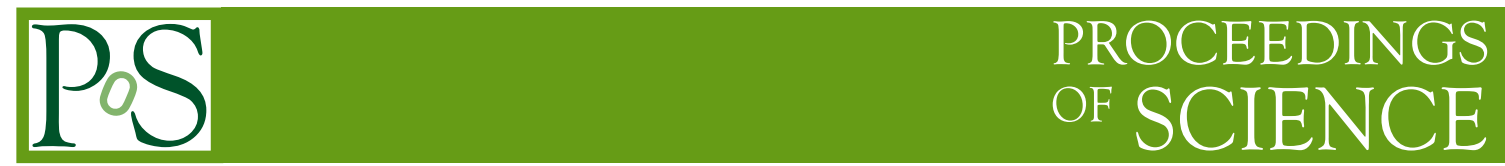

\title{
Gamma measurements at LHCb
}

\author{
Shu-Faye Cheung ${ }^{* \dagger}$ \\ University of Oxford \\ E-mail: shu-faye.cheung@physics.ox.ac.uk
}

Recent measurements of the CKM angle $\gamma$ from charged and neutral $\mathrm{B} \rightarrow \mathrm{DK}^{(*)}$ decays, with a variety of D final states, are presented. The measurements are performed using proton-proton collision data corresponding to an integrated luminosity of $3 \mathrm{fb}^{-1}$ collected at the LHCb experiment during Run I of the LHC. The results of a combination of LHCb $\gamma$ measurements are discussed. The combined value of $\gamma$ is $\left(70.9_{-8.5}^{+7.1}\right)^{\circ}$ and is the most precise measurement of $\gamma$ from a single experiment.

16th International Conference on B-Physics at Frontier Machines

2-6 May 2016

Marseille, France

* Speaker.

${ }^{\dagger}$ On behalf of the LHCb collaboration. 


\section{Introduction}

The direct measurement of the Cabibbo-Kobayashi-Maskawa matrix unitarity triangle angle $\gamma \equiv \arg \left(-V_{u d} V_{u b}^{*} / V_{c d} V_{c b}^{*}\right)$ in tree-level processes is insensitive to new physics contributions manifesting at loop level. Therefore, it serves as an excellent Standard Model benchmark, with a world average value of $\gamma=\left(73.2_{-7.0}^{+6.3}\right)^{\circ}$ [1]. In comparison, the world average indirect determination of $\gamma$, which is sensitive to new physics processes, is $\left(66.85_{-3.44}^{+0.94}\right)^{\circ}$. Although these measurements are compatible within the current uncertainties, driving the precision on the direct measurements to degree-level is necessary for the comparison to become meaningful.

The angle $\gamma$ can be measured using $\mathrm{B} \rightarrow \mathrm{DK}$ decays, where $\mathrm{D}$ is a superposition of $\mathrm{D}^{0}$ and $\overline{\mathrm{D}}^{0}$ mesons decaying to a common final state. The interference between $\mathrm{a} b \rightarrow \mathrm{u}$ and $\mathrm{b} \rightarrow \mathrm{c}$ transition provides access to $\gamma$. The two interfering Feynman diagrams in the $\mathrm{B}^{-}$decay are shown in Fig. 1 and are related by the ratio $\frac{A\left(\mathrm{~B}^{-} \rightarrow \overline{\mathrm{D}}^{0} \mathrm{~K}^{-}\right)}{A\left(\mathrm{~B}^{-} \rightarrow \mathrm{D}^{0} \mathrm{~K}^{-}\right)}=r_{B} e^{i\left(\delta_{B}-\gamma\right)}$, where $r_{B}$ is the magnitude ratio, $\delta_{B}$ is the strong-phase difference and $\gamma$ is the weak-phase difference. At LHCb, several different $\mathrm{B}$ decay modes have been used to measure $\gamma$. The same weak phase $\gamma$ is common to them all, while the hadronic parameters $r_{B}$ and $\delta_{B}$ depend on the specific B decay and can also be determined experimentally. The value of $r_{B}$ determines the sensitivity to $\gamma$, and is driven by the size of the participating CKM matrix elements and colour suppression factors. Depending on the D final state, $f$, the ratio between $\mathrm{D}^{0}$ and $\overline{\mathrm{D}}^{0}$ decay amplitude is $\frac{A\left(\mathrm{D}^{0} \rightarrow f\right)}{A\left(\overline{\mathrm{D}}^{0} \rightarrow f\right)}=r_{D} e^{i \delta_{D}}$, where $r_{D}$ and $\delta_{D}$ are the magnitude ratio and strong-phase difference, respectively.

In these proceedings, three recent measurements of $\gamma$ at LHCb using different B and D decays are presented, summarising the work in Refs. [2, 3, 4]. All three measurements were performed with proton-proton collision data at 7 and $8 \mathrm{TeV}$ corresponding to an integrated luminosity of $3 \mathrm{fb}^{-1}$. The result of the most recent LHCb $\gamma$ combination from B $\rightarrow$ DK-like decays is summarised [5].

\section{2. $\mathrm{B} \rightarrow \mathrm{DK}$ and $\mathrm{B} \rightarrow \mathrm{D} \pi$ decays with two- and four-body $\mathrm{D}$ final states}

In this analysis, $\mathrm{B} \rightarrow \mathrm{DK}$ and $\mathrm{B} \rightarrow \mathrm{D} \pi$ decays are analysed. The magnitude ratios for the two modes are $r_{B} \sim 0.1$ and $r_{B}^{D \pi} \sim 0.01$, respectively [6]. If the $\mathrm{D}$ meson decays to a $C P$ eigenstate, the values of $r_{D}$ and $\delta_{D}$ are simply determined by the $C P$ eigenvalue of the given final state. Here, the $C P$-even eigenstates $\mathrm{D} \rightarrow \pi^{+} \pi^{-}$and $\mathrm{K}^{+} \mathrm{K}^{-}$, where $r_{D}=1$ and $\delta_{D}=0$, are used. A similar fourbody mode, $\mathrm{D} \rightarrow \pi^{+} \pi^{-} \pi^{+} \pi^{-}$, is used in a $\gamma$ measurement for the first time. It has fractional $C P$ even content of $F_{+}^{4 \pi}=0.737 \pm 0.028$ [7], which dilutes interference effects by a factor $\left(2 F_{+}^{4 \pi}-1\right) \approx$
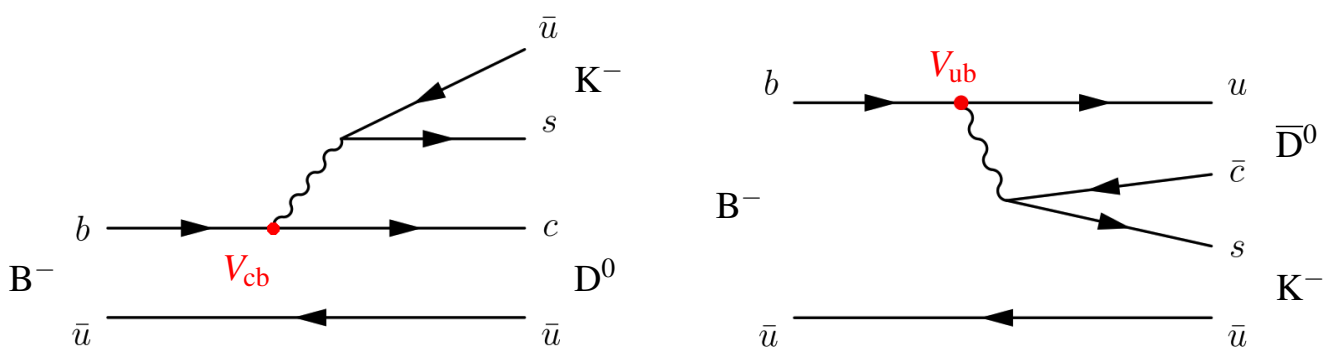

Figure 1: Feynman diagrams for (left) $\mathrm{B}^{-} \rightarrow \mathrm{D}^{0} \mathrm{~K}^{-}$and (right) $\mathrm{B}^{-} \rightarrow \overline{\mathrm{D}}^{0} \mathrm{~K}^{-}$amplitudes. 

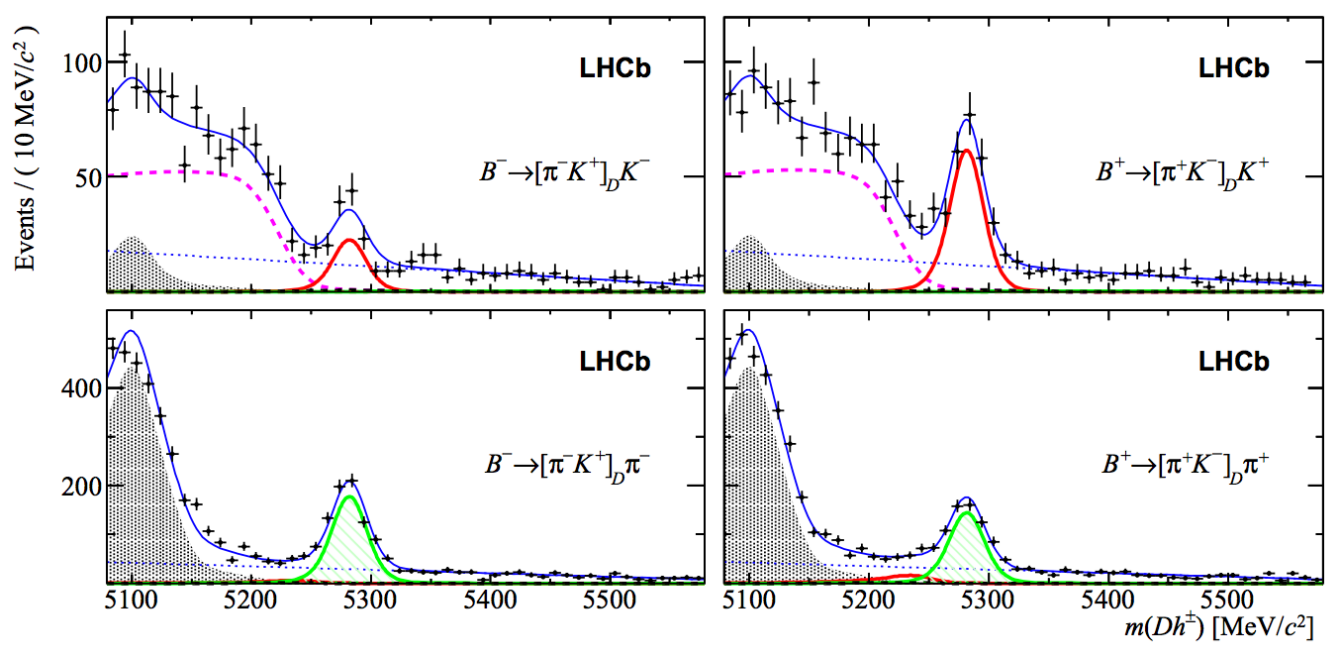

Figure 2: The $\mathrm{B}$ mass distributions of (top) $\mathrm{B} \rightarrow \mathrm{DK}$ and (bottom) $\mathrm{B} \rightarrow \mathrm{D} \pi$ candidates, with $\mathrm{D} \rightarrow \pi \mathrm{K}$. The distributions are separated by the (left) negative and (right) positive B meson charges. The (blue) total fit result is superimposed. The fit components are (red) $\mathrm{B} \rightarrow \mathrm{DK}$, (green) $\mathrm{B} \rightarrow \mathrm{D} \pi$, (dashed magenta) $\mathrm{B}_{\mathrm{s}}^{0} \rightarrow$ $\mathrm{DK}^{* 0}$, (dotted blue) combinatorial background and (black filled) other partially reconstructed backgrounds. Reproduced from [2].

0.5. The charge asymmetry is defined as:

$$
A_{h}^{f}=\frac{\Gamma\left(\mathrm{B}^{-} \rightarrow \mathrm{D}(\rightarrow f) \mathrm{h}^{-}\right)-\Gamma\left(\mathrm{B}^{+} \rightarrow \mathrm{D}(\rightarrow \bar{f}) \mathrm{h}^{+}\right)}{\Gamma\left(\mathrm{B}^{-} \rightarrow \mathrm{D}(\rightarrow f) \mathrm{h}^{-}\right)+\Gamma\left(\mathrm{B}^{+} \rightarrow \mathrm{D}(\rightarrow \bar{f}) \mathrm{h}^{+}\right)}
$$

where $\mathrm{h}$ refers to the pion or kaon produced in the B decay. With signal yields of $\mathscr{O}(1000)$ per D final state, $A_{K}^{K K}=0.087 \pm 0.020 \pm 0.008, A_{K}^{\pi \pi}=0.128 \pm 0.037 \pm 0.012$, and $A_{K}^{\pi \pi \pi \pi}=0.100 \pm$ $0.034 \pm 0.018$, where the first uncertainties are statistical and the second are systematic. The combined significance of $C P$ violation in the two-body modes is $5.0 \sigma$. Using the doubly-Cabibbo suppressed decay $\mathrm{D}^{0} \rightarrow \mathrm{K}^{+} \pi^{-}$, the presence of favoured and suppressed contributions to the two interfering decay paths leads to enhanced interference effects and larger asymmetries. The $\mathrm{K} \pi \pi \pi$ final state exhibits similar behaviour, apart from a coherence factor which dilutes the interference. The asymmetry for these two final states (so-called 'ADS' modes) is defined as:

$$
A_{\mathrm{ADS}(h)}^{\bar{f}}=\frac{\Gamma\left(\mathrm{B}^{-} \rightarrow \mathrm{D}(\rightarrow \bar{f}) \mathrm{h}^{-}\right)-\Gamma\left(\mathrm{B}^{+} \rightarrow \mathrm{D}(\rightarrow f) \mathrm{h}^{+}\right)}{\Gamma\left(\mathrm{B}^{-} \rightarrow \mathrm{D}(\rightarrow \bar{f}) \mathrm{h}^{-}\right)+\Gamma\left(\mathrm{B}^{+} \rightarrow \mathrm{D}(\rightarrow f) \mathrm{h}^{+}\right)}
$$

and is measured to be $A_{\mathrm{ADS}(\mathrm{K})}^{\pi \mathrm{K}}=-0.403 \pm 0.056 \pm 0.011$ and $A_{\mathrm{ADS}(\pi)}^{\pi \mathrm{K}}=0.100 \pm 0.031 \pm 0.009$. The significance of $C P$ violation in $A_{\mathrm{ADS}(\mathrm{K})}^{\pi \mathrm{K}}$ is $8.0 \sigma$ and represents the first time that $C P$ violation is observed in a single $\mathrm{B} \rightarrow \mathrm{Dh}$ decay mode with greater than $5 \sigma$ significance. The $\mathrm{B}$ mass distributions of $\mathrm{B}^{ \pm} \rightarrow \mathrm{D}\left(\rightarrow \pi^{ \pm} \mathrm{K}^{\mp}\right) \mathrm{h}^{ \pm}$candidates are shown in Fig. 2, where the charge asymmetries are visually observable as differences in peak heights between the $\mathrm{B}^{-}$and $\mathrm{B}^{+}$data. In the four-body ADS modes, the asymmetries are $A_{\mathrm{ADS}(\mathrm{K})}^{\pi \mathrm{K} \pi \pi}=-0.313 \pm 0.102 \pm 0.038$ and $A_{\mathrm{ADS}(\pi)}^{\pi \mathrm{K} \pi \pi}=$ $0.023 \pm 0.048 \pm 0.005$. As expected, the presence of a coherence factor causes the size of the interference, and hence the asymmetry, to be smaller than in the two-body case. An exhaustive list of the observables measured is provided in Ref. [2], and they are generally found to be consistent with expectation from existing knowledge of the underlying physics parameters. 


\section{3. $\mathrm{B}^{0} \rightarrow \mathrm{DK}^{* 0}$ decays with $\mathrm{D} \rightarrow \mathrm{K}_{\mathrm{S}}^{0} \pi^{+} \pi^{-}$and $\mathrm{K}_{\mathrm{S}}^{0} \mathrm{~K}^{+} \mathrm{K}^{-}$}

$\mathrm{B}^{0} \rightarrow \mathrm{DK}^{*}(892)^{0}$ decays can also be used to measure $\gamma$. The interference effects in this decay are 3-4 times larger than in $\mathrm{B} \rightarrow$ DK due to colour suppression in both interfering amplitudes of the $\mathrm{B}^{0} \rightarrow \mathrm{DK}^{* 0}$ decay. The hadronic parameters associated with the $\mathrm{B}^{0} \rightarrow \mathrm{DK}^{* 0}$ decay, $r_{B^{0}}$ and $\delta_{B^{0}}$, are measured in the range $\left|m(\mathrm{~K} \pi)-m\left(\mathrm{~K}^{*}(892)^{0}\right)_{\mathrm{PDG}}\right|<50 \mathrm{MeV} / c^{2}$ and $\left|\cos \theta^{*}\right|>0.4$, where $\cos \theta^{*}$ is the cosine of the angle between the kaon and the $\mathrm{B}$ in the $\mathrm{K}^{* 0}$ rest frame. The charge of the kaon from the $\mathrm{K}^{* 0}$ decay tags the flavour of the $\mathrm{B}^{0}$ unambiguously. The multibody $\mathrm{D} \rightarrow \mathrm{K}_{\mathrm{S}}^{0} \mathrm{~h}^{+} \mathrm{h}^{-}$ decay, $\mathrm{h}=\{\mathrm{K}, \pi\}$, proceeds via several intermediate resonances which interfere, causing the value of $\delta_{D}$ to vary continuously in the two-dimensional $\mathrm{D} \rightarrow \mathrm{K}_{\mathrm{s}}^{0} \mathrm{~h}^{+} \mathrm{h}^{-}$Dalitz plot. This manifests as local asymmetries in different parts of the Dalitz plots from $\mathrm{B}^{0}$ and $\overline{\mathrm{B}}^{0}$ decays (see Fig. 3). There are two methods to account for the variation in $\delta_{D}$, known as the 'model-dependent' and 'modelindependent' methods. Both are used to measure $\gamma$ at $\mathrm{LHCb}$ and are described below.

\subsection{The model-dependent method}

In the model-dependent method, an amplitude model for the $\mathrm{D}^{0} \rightarrow \mathrm{K}_{\mathrm{S}}^{0} \pi^{+} \pi^{-}$decay is used to provide the magnitude and strong phase of the $\mathrm{D}^{0}$ decay amplitude at each point of the Dalitz plot. Assuming no $C P$ violation in the $\mathrm{D}$ meson decay, the $\overline{\mathrm{D}}^{0}$ decay amplitude can be inferred, allowing $\delta_{D}$ to be calculated. The $2010 \mathrm{BaBar}$ amplitude model is used [8]. The measured $C P$ violation observables are defined as $x_{ \pm}=r_{B^{0}} \cos \left(\delta_{B^{0}} \pm \gamma\right)$ and $y_{ \pm}=r_{B^{0}} \sin \left(\delta_{B^{0}} \pm \gamma\right)$. Using approximately $90 \mathrm{~B}^{0} \rightarrow \mathrm{D}\left(\rightarrow \mathrm{K}_{\mathrm{s}}^{0} \pi^{+} \pi^{-}\right) \mathrm{K}^{* 0}$ decays, the results are $x_{+}=0.05 \pm 0.24 \pm 0.04 \pm 0.01, x_{-}=$ $-0.15 \pm 0.14 \pm 0.03 \pm 0.01, y_{+}=-0.65_{-0.33}^{+0.24} \pm 0.08 \pm 0.01$ and $y_{-}=0.25 \pm 0.15 \pm 0.06 \pm 0.01$ [4] where the first uncertainties are statistical, the second are systematic and the third are modelrelated uncertainties. When all sources of uncertainties are combined, quoting the solution where $\gamma \in\left[0^{\circ}, 180^{\circ}\right]$, the physics parameters are $r_{B^{0}}=0.39 \pm 0.13, \delta_{B^{0}}=\left(197_{-20}^{+24}\right)^{\circ}$ and $\gamma=\left(80_{-22}^{+21}\right)^{\circ}$.

\subsection{The model-independent method}

Strong-phase difference parameters of the D meson have been measured by the CLEO experiment using quantum-correlated $\psi(3770) \rightarrow \mathrm{D}^{0} \overline{\mathrm{D}}^{0}$ decays [9]. These strong-phase parameters, measured in binned regions of the Dalitz plot, provide direct access to $\delta_{D}$ and have been measured for both $\mathrm{D} \rightarrow \mathrm{K}_{\mathrm{S}}^{0} \pi^{+} \pi^{-}$and $\mathrm{K}_{\mathrm{S}}^{0} \mathrm{~K}^{+} \mathrm{K}^{-}$. The same sample of $\mathrm{D} \rightarrow \mathrm{K}_{\mathrm{s}}^{0} \pi^{+} \pi^{-}$as before is used, with the addition of approximately seven $\mathrm{K}_{\mathrm{S}}^{0} \mathrm{~K}^{+} \mathrm{K}^{-}$candidates. The results are $x_{+}=0.05 \pm 0.35 \pm 0.02$, $x_{-}=-0.31 \pm 0.20 \pm 0.04, y_{+}=-0.81 \pm 0.28 \pm 0.06$ and $y_{-}=0.31 \pm 0.21 \pm 0.05$ [3], where the first uncertainties are statistical and the second are systematic. The uncertainties associated with the CLEO measurements are contained within the statistical uncertainties and are estimated to be 0.02 for $x_{ \pm}$and 0.05 for $y_{ \pm}$. When all sources of uncertainties are combined, the physics parameters are $r_{B^{0}}=0.56 \pm 0.17, \delta_{B^{0}}=\left(204_{-20}^{+21}\right)^{\circ}$ and $\gamma=(70 \pm 20)^{\circ}$.

The likelihood contours on $x_{ \pm}$and $y_{ \pm}$for the statistical uncertainties from the two methods are shown in Fig. 4. The results from the two methods are consistent and cannot be combined because the same $\mathrm{D} \rightarrow \mathrm{K}_{\mathrm{S}}^{0} \pi^{+} \pi^{-}$dataset is used. 

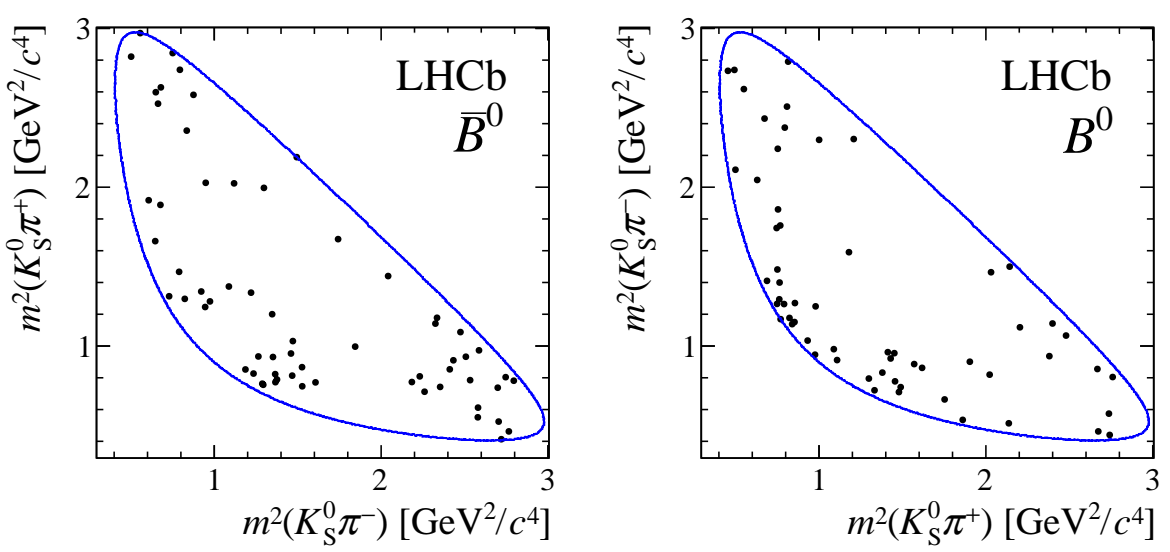

Figure 3: Dalitz plots of $\mathrm{D} \rightarrow \mathrm{K}_{\mathrm{S}}^{0} \pi^{+} \pi^{-}$signal candidates from (left) $\overline{\mathrm{B}}^{0} \rightarrow \mathrm{D} \overline{\mathrm{K}}^{* 0}$ and (right) $\mathrm{B}^{0} \rightarrow \mathrm{DK}^{* 0}$ decays. The blue line denotes the kinematic boundary. Reproduced from [3].
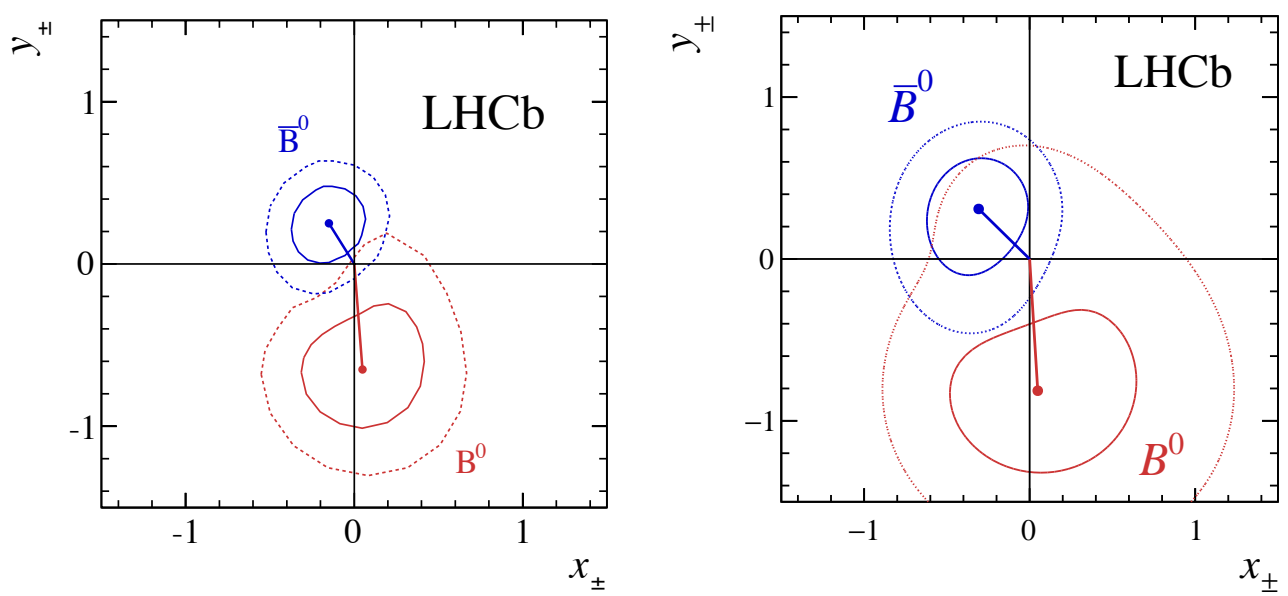

Figure 4: The (solid) $1 \sigma$ and (dotted) $2 \sigma$ likelihood contours corresponding to the statistical uncertainties on the observables $x_{ \pm}$and $y_{ \pm}$in the (left) model-dependent and (right) model-independent analyses of $\mathrm{B}^{0} \rightarrow$ $\mathrm{DK}^{* 0}, \mathrm{D} \rightarrow \mathrm{K}_{\mathrm{S}}^{0} \mathrm{~h}^{+} \mathrm{h}^{-}$decays. The central values are indicated by the points. Reproduced from [3, 4].
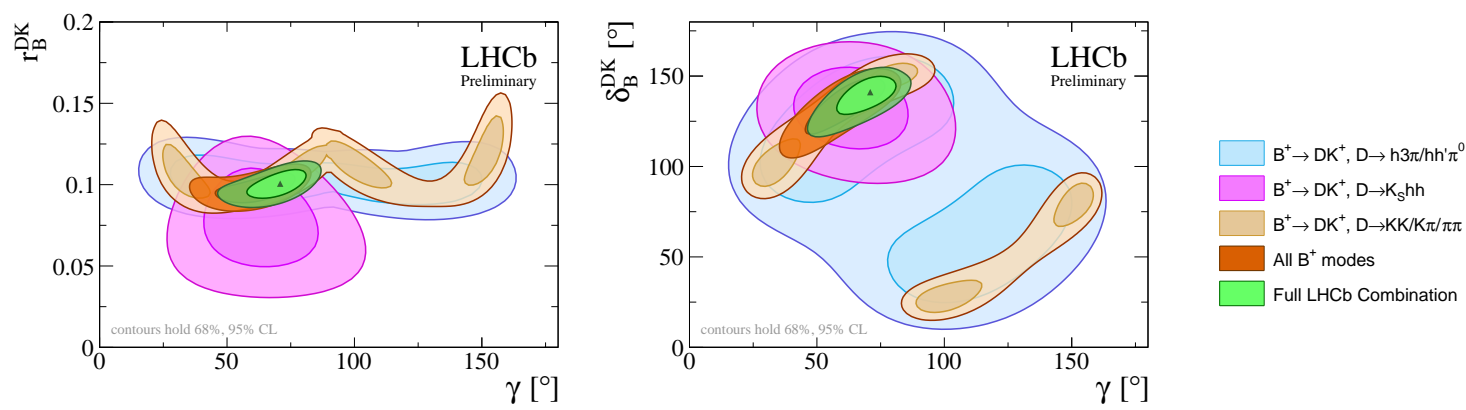

Figure 5: Two-dimensional likelihood contours in (left) $\left(\gamma, r_{B}\right)$ and (right) $\left(\gamma, \delta_{B}\right)$, corresponding to one and two standard deviations. Reproduced from [5]. 


\section{Combination of results}

The latest $\mathrm{LHCb}$ combination of $\gamma$ measurements using $\mathrm{B} \rightarrow$ DK-like decays is presented below. The $\mathrm{B}$ decays are $\mathrm{B}^{+} \rightarrow \mathrm{DK}^{+}, \mathrm{B}^{0} \rightarrow \mathrm{DK}^{+} \pi^{-}, \mathrm{B}^{0} \rightarrow \mathrm{DK}^{* 0}, \mathrm{~B}^{+} \rightarrow \mathrm{DK}^{+} \pi^{+} \pi^{-}$and $\mathrm{B}_{\mathrm{s}}^{0} \rightarrow$ $\mathrm{D}_{\mathrm{s}}^{-} \mathrm{K}^{+}$. The complete list of inputs can be found in Ref. [5]. The contribution of different analysis modes to the combination is demonstrated in Fig. 5, in which the two-dimensional likelihood contours on $\left(\gamma, r_{B}\right)$ and $\left(\gamma, \delta_{B}\right)$ for $\mathrm{B} \rightarrow$ DK measurements are drawn separately for different classes of D final states. The combined values of the hadronic parameters are $r_{B}=0.1006_{-0.0060}^{+0.0059}$, $\delta_{B}=\left(141.1_{-7.7}^{+6.1}\right)^{\circ}, r_{B^{0}}=0.217_{-0.048}^{+0.044}$ and $\delta_{B^{0}}=\left(189_{-20}^{+24}\right)^{\circ}$, and are consistent with expectations. The combined value is $\gamma=\left(70.9_{-8.5}^{+7.1}\right)^{\circ}$ and is consistent with, and approximately a factor of two better than, the results obtained by each of the B-factory experiments.

\section{Summary}

The combined value of $\gamma$ from many LHCb measurements is $\gamma=\left(70.9_{-8.5}^{+7.1}\right)^{\circ}$, which is the most precise determination of $\gamma$ from a single experiment. Improved precision from LHCb can be expected in the coming years when data from Run II of the LHC is analysed and new B and D decay modes are exploited. In the near future, a combination of results including $\mathrm{B} \rightarrow \mathrm{D} \pi$ decays will be presented.

\section{References}

[1] CKMfitTer Group collaboration, J. Charles et al., CP violation and the CKM matrix: Assessing the impact of the asymmetric B factories, Eur. Phys. J. C41 (2005) 1-131, [hep-ph/ 0406184 ].

[2] LHCB collaboration, R. Aaij et al., Measurement of CP observables in $B^{ \pm} \rightarrow D K^{ \pm}$and $B^{ \pm} \rightarrow D \pi^{ \pm}$ with two- and four-body D meson decays, Phys. Lett. B760 (2016) 117, [1603.08993].

[3] LHCB collaboration, R. Aaij et al., Model-independent measurement of the CKM angle $\gamma$ using $B^{0} \rightarrow D K^{* 0}$ decays with $D \rightarrow K_{S}^{0} \pi^{+} \pi^{-}$and $K_{S}^{0} K^{+} K^{-}, J H E P 06$ (2016) 131, [1604.01525].

[4] LHCB collaboration, R. Aaij et al., Measurement of the CKM angle $\gamma$ using $B^{0} \rightarrow D K^{* 0}$ with $D \rightarrow K_{S}^{0} \pi^{+} \pi^{-}$decays, submitted to JHEP, LHCb-PAPER-2016-007, [1605.01082].

[5] LHCB collaboration, $L H C b \gamma$ combination update from $B \rightarrow D K X$ decays, LHCb-CONF-2016-001.

[6] M. Gronau and J. L. Rosner, Final state interaction effects on $\gamma$ from B $\rightarrow$ DK, Phys. Lett. B439 (1998) 171-175, [hep-ph/9807447].

[7] S. Malde, C. Thomas, G. Wilkinson, P. Naik, C. Prouve, J. Rademacker et al., First determination of the $C P$ content of $D \rightarrow \pi^{+} \pi^{-} \pi^{+} \pi^{-}$and updated determination of the $C P$ contents of $D \rightarrow \pi^{+} \pi^{-} \pi^{0}$ and $D \rightarrow K^{+} K^{-} \pi^{0}$, Phys. Lett. B747 (2015) 9-17, [1504.05878].

[8] BABAR collaboration, P. del Amo Sanchez et al., Evidence for direct CP violation in the measurement of the Cabibbo-Kobayashi-Maskawa angle $\gamma$ with $B^{\mp} \rightarrow D^{(*)} K^{(*) \mp}$ decays, Phys.Rev.Lett. 105 (2010) 121801, [1005.1096].

[9] CLEO collaboration, J. Libby et al., Model-independent determination of the strong-phase difference between $D^{0}$ and $\bar{D}^{0} \rightarrow K_{\mathrm{S}, \mathrm{L}}^{0} h^{+} h^{-}(h=\pi, K)$ and its impact on the measurement of the CKM angle $\gamma / \phi_{3}$, Phys.Rev. D82 (2010) 112006, [1010.2817]. 